\title{
Im Grenzgelände von Jugendhilfe und Ausländerrecht
}

\section{Die Inobhutnahme minderjähriger unbegleiteter Flüchtlinge ist in Deutschland ein ungelöstes Problem}

\author{
Harald Löhlein und Norbert Struck
}

Migration and youth welfare have actually several points of contact. A specifically delicate one is the concern for underage refugees who are over 16 years of age living in Germany and their right to custody. These cases show in an exemplary way some of the difficulties of a policy that is oriented towards the best of the child and does not want to be forced by questions of policies concerned with foreigners.

Les migrations et l'Aide à la jeunesse ont en fait plusieurs points en commun. Un d'entre eux, particulièrement délicat, est le problème des jeunes mineurs réfugiés de plus de 16 ans, résidant en Allemagne et leur droit à bénéficier d'une garde. Ces cas montrent de manière exemplaire certaines des difficultés que rencontrent les politiques qui visent le bien être des enfants qui ne veulent pas être mêlées à des questions de politique d'accueil des étrangers.

Harald Löhlein ist Referent für Flüchtlingshilfe, Norbert Struck ist Referent für Jugendhilfe beim Paritätischen Wohlfahrtsverband Gesamtverband e. V. E-Mail jugendhilfe@paritaet.org
Zwischen den Themen Migration und Jugendhilfe gibt es manche Berührungen. Eine besonders sensible Schnittstelle stellt die Situation von über 16-jährigen minderjährigen unbegleiteten Flüchtlingen in Deutschland und ibr Recht auf Inobhutnabme dar. An ibr zeigen sich exemplarisch einige Schwierigkeiten einer Politik, die am Kindeswohl orientiert ist und sich nicht ausländerpolitisch in die Knie zwingen lassen will.

1.

Seit Jahren sind die Zahlen der unbegleiteten minderjährigen Flüchtlingen rückläufig, ebenso wie die Zahl der Asylbewerber insgesamt. So sank die Zahl der jugendlichen unbegleiteten Asylbewerber (Erstantragsteller) zwischen 2002 und 2005 von 873 auf 331, die Zahl der unbegleiteten minderjährigen Flüchtlingen, die von den zuständigen Jugendämtern in Obhut genommen wurden, im gleichen Zeitraum von 1.441 auf 602. Ihre Schutzquote, also der Anteil derer, die entweder als Asylberechtigte anerkannt wurde oder subsidiären Schutz erhielten, lag in der Vergangenheit in der Regel zwar höher, als bei den Asylbewerbern insgesamt, war aber immer noch gering (2005: 5,65\% Anmerkung 1) Die Hoffnung, die geringeren Zahlen hätten nun dazu geführt, dass es insgesamt zu einem angemesseneren, die spezifischen Schutzbedürfnisse von Minderjährigen ernst nehmenden Umgang mit unbegleiteten minderjährigen Flüchtlingen gekommen wäre, haben sich nicht erfüllt.

2.

Im Jahre 1992 hat die Bundesregierung die UN-Kinderrechtskonvention nur un- ter Vorbehalt ratifiziert: Keine Bestimmung der UN-Kinderrechtskonvention sollte dahingehend ausgelegt werden können, dass sie das Recht der Bundesrepublik beschränkte, Gesetze und Verordnungen über die Einreise oder den Aufenthalt von Ausländern zu erlassen. Seither kann Rechtssetzung international oder national geschehen, wie sie will, die meisten Innenminister von Bund und Land gehen davon aus, dass alle Neuerungen per se mit dem von ihnen angewandten Ausländerrecht »im Einklang “ sind und dass für sie und die ihnen unterstellten Behörden kein Zwang zur Veränderung aus Rechtssetzungen erwachsen kann, bei denen sie nicht die Federführung haben. Unter dieser Praxis haben in Deutschland insbesondere die minderjährigen unbegleiteten Flüchtlinge zu leiden, die nach Einschätzung der Behörden das 16. Lebensjahr vollendet haben. Während bei den unter 16-Jährigen in aller Regel die Normen des Kinder- und Jugendhilfegesetzes (SGB VIII) angewandt werden und Einrichtungen der Kinderund Jugendhilfe zu ihrer Betreuung in Anspruch genommen werden, wird bei den 16- bis 18-Jährigen oft das Verteilungsund Unterbringungsverfahren des Ausländerrechts zur Anwendung gebracht. Seit ihrer Gründung im Jahr 1995 thematisiert die National Coalition (NC) die Notwendigkeit zur Rücknahme der Ratifizierungsvorbehalte insbesondere im Hinblick auf die Situation unbegleiteter Flüchtlinge. Auch der UN-Ausschuss, der sich mit der Umsetzung der UN-Kinderrechtskonvention befasst, hat dieses Thema immer wieder aufgegriffen. (2) Ebenso gab es verschiedene Initiativen der Kinderkommission des Deutschen Bundestages zur Zurücknahme des Vorbehalts. Bisher sind diese Initiativen im Zuständigkeitsgewirr von Bund und Ländern versandet. 
3.

Das Recht der Inobhutnahme im Rahmen des Kinder- und Jugendhilferechts wurde im Jahre 2005 durch das Kinderund Jugendhilfeweiterentwicklungsgesetz (KICK) gestrafft und neu geregelt $(\mathbb{S} 42$ SGB VIII - Anmerkung 3). Erstmals wurde in diesem Rahmen auch die Situation unbegleiteter minderjähriger Flüchtlinge explizit Gegenstand der Regelung:

»(1) Das Jugendamt ist berechtigt und verpflichtet, ein Kind oder einen Jugendlichen in seine Obhut zu nehmen wenn [...]

3. ein ausländisches Kind oder ein ausländischer Jugendlicher unbegleitet nach Deutschland kommt und sich weder Personensorge noch Erziehungsberechtigte im Inland aufhalten. [...]

(3) [...] Im Fall des Absatzes 1 Satz 1 Nr. 3 ist unverzüglich die Bestellung eines Vormundes oder Pflegers zu veranlassen.

(4) Die Inobhutnahme endet mit

1. der Übergabe des Kindes oder Jugendlichen an die Personensorge- oder Erziehungsberechtigten,

2. der Entscheidung über die Gewährung von Hilfen nach dem Sozialgesetzbuch."

In der Begründung zum Regierungsentwurf heißt es hierzu lediglich: "Schließlich wurde auch die vorläufige Versorgung unbegleiteter Minderjähriger, die bereits derzeit auf der Grundlage von $\mathbb{S}$ 42 erfolgt, mit ihren spezifischen Anforderungen geregelt. " Deutlicher im Hinblick auf die Motivation zur Neuregelung wird allerdings Reinhard Wiesner in seinem Kommentar zu $\mathbb{\$}$ 42: »Im Rahmen des KICK wurde die Regelung ausdrükklich auch auf die Einreise eines ausländischen Kindes oder Jugendlichen ausgedehnt, das bzw. der unbegleitet nach Deutschland kommt und hier weder mit einem Personensorgeberechtigten noch mit einem Erziehungsberechtigten zusammentrifft [...] Der Gesetzgeber reagiert damit auf die Kritik des UN-Kinderrechteausschusses. «(Wiesner, 2006, $\mathbb{S} 42$ Rz.16)

Diesen Zusammenhang stellt auch der »Nationale Aktionsplan für ein kindgerechtes Deutschland 2005-2010« der Bundesregierung her. Unter Punkt 2.6.2 heißt es dort:

»Die Bundesregierung wird sich dafür einsetzen, dass für alle betroffenen unbegleiteten schutzsuchenden Kinder und Ju- gendlichen ein so genanntes Clearingverfahren durchgeführt wird. $\mathrm{Zu}$ diesem Zweck sieht bereits der Entwurf eines Gesetzes zur Weiterentwicklung der Kinderund Jugendhilfe die Erstversorgung eines unbegleiteten minderjährigen Flüchtlings im Rahmen der Inobhutnahme durch das Jugendamt vor. In dem Verfahren soll auch geklärt werden, ob eine Rückreise in das Heimatland ohne erhebliche Gefahren möglich ist, ob eine Familienzusammenführung in einem Drittland in Frage kommt, ob ein Asylantrag gestellt oder ein Bleiberecht aus humanitären Gründen angestrebt werden soll.

Sie wird darauf hinwirken, dass entsprechend der Gesetzeslage auch auf sich alleine gestellten 16- und 17-jährigen ausländischen Kindern so schnell wie möglich nach der Einreise ein Vormund zur Seite gestellt wird. Im oben genannten Gesetzgebungsverfahren wird dazu im Fall der Inobhutnahme durch das Jugendamt ausdrücklich die Verpflichtung geregelt, die Bestellung eines Vormunds oder Pflegers für eingereiste unbegleitete Kinder und Jugendliche zu veranlassen. «

Für alle, die sich seit Jahren für die Rechte und den Schutz unbegleiteter minderjähriger Flüchtlinge einsetzen, schien damit endlich eine Lösung gefunden worden zu sein. Die Norm anerkennt die Situation, in der diese jungen Menschen sich in einem fremden Land befinden als eine per se das Kindeswohl gefährdende an: "Mit der Neuregelung ist der unter der Geltung des $\ 42$ SGB VIII a.F. bestehende Diskurs, ob die Regelung über die Inobhutnahme auf die Fallgestaltung der unbegleiteten minderjährigen Flüchtlinge überhaupt (analog) anwendbar ist, bedeutungslos geworden. Mit Blick auf den eindeutigen Wortlaut der Neuregelung wird den Jugendämtern bei Vorliegen ihrer tatbestandlichen Voraussetzungen eine stringente Handlungspflicht auferlegt. « (4)

Der $\int 89 \mathrm{~d}$ SGB VIII regelt auch, dass der zuständige örtliche Träger nicht auf den Kosten dieser Inobhutnahme hängen bleibt, sondern sie vom zuständigen Land erstattet bekommt.

Und die Komplikationen, die sich bei der Anwendung von Jugendhilferecht ansonsten durch die Kaskade von Rechtstiteln zum Aufenthalt gibt, stellen sich bei der Gewährung von Hilfen für unbegleitete minderjährige Flüchtlinge sowieso nicht, da der Aufenthaltstitel des leis- tungsberechtigten Vormunds ja in der Regel unstrittig und unkompliziert ist.

\section{4.}

Und dennoch zeigt sich, dass in der Praxis teilweise die alten Probleme fortbestehen:

- Der Zugang zur Inobhutnahme. Zwar ist die Verpflichtung des Jugendamtes zur Inobhutnahme eindeutig. Aber wie kommt der Jugendliche zum Jugendamt? Der Sache nach muss jede Behörde, bei der sich ein minderjähriger unbegleiteter Flüchtling meldet, das Jugendamt am Ort verständigen, damit dies umgehend die Inobhutnahme durchführen kann. Zuständig ist immer das Jugendamt an dem Ort, an dem der junge Mensch sich aufhält ( $\mathbb{S} 87$ SGB VIII). Die Frage, ob der junge Mensch minderjährig ist oder nicht, entscheidet sich entweder anhand vorgelegter $\mathrm{Pa}$ piere oder aber anhand seiner Selbstauskunft. In der Praxis zeigt sich aber, dass Ausländerbehörden oder Polizei diese Verpflichtung oft nicht umsetzen. Nicht immer geschieht dies offenbar aus Unkenntnis der Rechtsnormen. Teilweise wird diese Verpflichtung bewusst ignoriert. So droht etwa nach wie vor unbegleiteten minderjährigen Jugendlichen die Zurückweisung oder Zurückschiebung an der Grenze, da nach Ansicht der Bundesregierung die Verpflichtung zur Information der Jugendämter (Inobhutnahme) erst bei denjenigen Kindern und Jugendlichen gegeben ist, die bereits nach Deutschland eingereist sind, nicht aber bei denjenigen, denen von den Grenzbehörden die Einreise verweigert wird. (5)

- Die Einsetzung von Vormündern. In $\mathbb{S}$ 42 Abs. 3 SGB VIII ist als Aufgabe des Jugendamtes klar die unverzügliche Veranlassung der Bestellung eines Vormunds oder Pflegers benannt. In aller Regel geht es in der Praxis um die Bestellung eines Vormunds, da die elterliche Sorge voll ersetzt werden muss $(\mathbb{S}$ 1773 f BGB), weil die elterliche Sorge ruht ( $\mathbb{1 6 7 4}$ BGB). Der Vormund ist auf das Wohl des Jugendlichen verpflichtet und muss dementsprechende $\mathrm{Maßnahmen} \mathrm{ergreifen.} \mathrm{Er} \mathrm{ist} \mathrm{vom} \mathrm{Fa}$ miliengericht nach Anhörung des Jugendamts auszuwählen ( $\mathbb{1 7 7 9}$ BGB). Das Recht sieht zwar die Benennung 
natürlicher Personen als Regelfall an, aber in der Praxis wird zumeist ein Amtsvormund, also eine Mitarbeiterin des Jugendamts eingesetzt $(\mathbb{S} 1791 \mathrm{~b}$ BGB). Gegebenenfalls kann noch ein Anwalt zusätzlich als Pfleger bestellt werden für die Interessenvertretung im ausländer- und asylrechtlichen Verfahren. Im Hinblick auf alle ausländerrechtlichen Klärungsbedarfe, Entscheidungen und Maßnahmen muss der Vormund von den Behörden informiert werden, sonst kann er seinen Verpflichtungen nicht nachkommen.

- Verteilungsprobleme. Häufig wird ein Vormund für unbegleitete Minderjährige aber erst nach der Asylantragstellung eingeschaltet, wenn der Jugendliche möglicherweise schon im Rahmen des im Asylverfahrensgesetz festgeschriebenen Verteilverfahrens, wie es für Asylbewerber generell vorgesehen ist, in ein anderes Bundesland oder eine andere Kommune umverteilt worden ist. Wichtige Fragen, wie die Feststellung des Alters oder die Frage, ob überhaupt ein Asylantrag gestellt werden sollte, sind dann schon geklärt. Oftmals sind die jungen Menschen dann zunächst in Zentralen Aufnahmeeinrichtungen der Länder oder in Gemeinschaftsunterkünften untergebracht, nicht aber in Einrichtungen, die eine kindgerechte Betreuung gewährleisten.

- Standards der Inobhutnahme. Die Inobhutnahme hat als sozialpädagogische Krisenintervention nach fachlichen Standards zu erfolgen. Ein Qualität sicherndes Instrument des SGB VIII, das dies gewährleisten soll, ist die Erlaubniserteilung nach $\int 45$ durch das Landesjugendamt. Derzeit wird zwischen Bund und Ländern an einem "Handlungsleitfaden « gearbeitet, bei dem die Innenpolitiker eine Interpretation durchsetzen möchten, nach der Aufnahmeeinrichtungen unter ihrer Aufsicht den Befreiungstatbestand des $\mathbb{4} 45$ Abs. 1 Nr. 3 SGB VIII erfüllen. Damit würden die Intentionen der Neuregelung des KICK wieder ins Gegenteil verkehrt! Zum Glück ist eine solche Interpretation rechtlich nicht haltbar, denn die Erlaubnisbefreiung gilt explizit nur für solche Einrichtungen, die »außerhalb der Jugendhilfe liegende Aufgaben für Kinder und Jugendliche « wahrnehmen. Eine Inobhutnahme ist aber zweifelsfrei eine innerhalb der Jugendhilfe liegende Aufgabe - und deren Wahrnehmung in einer Einrichtung verlangt eine Betriebserlaubnis und diese wiederum den Einsatz von Fachkräften nach $\mathbb{} \$ 2$ SGB VIII. Es spricht vieles dafür, spezialisierte Clearingstellen für die Begleitung und Betreuung unbegleiteter Minderjähriger im Rahmen der Inobhutnahme vorzuhalten, wenn dies bedeutet, dass die Aufgaben des Klärens und der Organisation von Hilfe und Unterstützung durch besonders erfahrene Fachkräfte wahrgenommen werden und routinemäßig Sprachkompetenzen verfügbar sind. Basis der Arbeit solcher Clearingstellen müssen aber fachliche Standards und eine Betriebserlaubnis nach $\int 45$ SGB VIII sein. Es kann nicht angehen, dass lediglich Erstaufnahmeeinrichtungen $\mathrm{zu}$ »besonderen Aufnahmeeinrichtungen « umdeklariert werden und dann suggeriert wird, den rechtlichen Erfordernissen sei Rechnung getragen! Die neue Fassung des $\$ 42$ SGB VIII lässt bei der Inobhutnahme auch eine Unterbringung in einer »sonstigen Wohnform«zu. Diese Option sollte niedrigschwellige Organisationsformen bei Jugendlichen zulassen, die sich einer Einrichtung gleich wieder entziehen würden, sie ist keineswegs geeignet als Legitimation für die Unterbringung in einer Aufnahmeeinrichtung.

- Abschiebehaft. Soweit unbegleitete minderjährige Flüchtlinge nach der Einreise in Abschiebehaft genommen werden, gibt es in der Regel keine Inobhutnahme durch die Jugendämter und keine Regelung der gesetzlichen Vertretung. Zwar ist gemäß $\int 62$ Abs. 2 AufenthG auch bei Minderjährigen die Anordnung von Sicherungshaft möglich. Es ist aber auf jeden Fall vorab zu klären, ob es nicht ein milderes Mittel als die Abschiebehaft gibt. Dies könnte insbesondere die Unterbringung in einer Clearingstelle sein. Daher ist wichtig, dass auch in den Fällen, in denen unbegleitete minderjährige Flüchtlinge in Abschiebehaft genommen werden, das Jugendamt zuständig ist für die Durchführung der Schutzmaßnahmen.

- Transformation in Jugendhilfeleistung. Eine wesentliche Aufgabe des Vormundes ist es, den Hilfebedarf im Einzelfall zu klären. Ein Amtsvormund, der die elterliche Sorge innehat, sollte hier sorgfältig vorgehen und sich die Hilfe organisieren, die er braucht, um sein Amt verant- wortungsvoll auszuüben. Erst wenn diese Hilfeplanung erfolgreich abgeschlossen worden ist, kann die Inobhutnahme beendet werden. Keinesfalls endet die Inobhutnahme schon mit der Bestellung des Vormunds, denn mit dieser Bestellung ist ja noch keine der weiteren Aufgaben der Inobhutnahme gelöst. (6) Nach $\ 42$ Abs. 4 SGB VIII setzt die Beendigung einer Inobhutnahme eine Entscheidung über die Gewährung von Hilfen nach dem Sozialgesetzbuch voraus, also eine abgeschlossene Hilfeplanung.

- Probleme der Altersfeststellung. Die Altersfeststellung erfolgt, da die Jugendlichen in der Regel keine Dokumente vorlegen können, aus denen sich das Alter zweifelsfrei ergibt, bei den Ausländerbehörden teilweise immer noch nach Augenschein. Der derzeit vorliegende Entwurf für die Änderung des Aufenthaltsgesetzes sieht hier eine weitere Verschärfung vor. So sollen zukünftig bei Ausländern, die das 14. Lebensjahr vollendet haben u. a. "Messungen und ähnliche Maßnahmen, einschließlich körperlicher Eingriffe, die von einem Arzt nach den Regeln der ärztlichen Kunst nach den zum Zwecke der Feststellung des Alters « (7) vorgenommen werden können, wenn kein Nachteil für die Gesundheit des Ausländers zu befürchten ist. Zweifel an der Vollendung des 14. Lebensjahres sollen dabei zu Lasten des Ausländers gehen. Da minderjährige Flüchtlinge in der Regel kaum die Kosten für ein Gutachten zur Altersfeststellung aufbringen können, sind sie demzufolge faktisch der Einschätzung durch die Behörden ausgeliefert.

\section{Fazit}

Die internationalen und nationalen Normen können noch so klar sein, die Schutzbedürftigkeit Minderjähriger, die nach Flucht oder Vertreibung in ein fremdes Land, mit zumeist fremder Kultur und Sprache kommen, noch so evident, die Rechtsumsetzung bleibt ein Problem, dem weiter die politische Aufmerksamkeit gebührt. Es ist schwer nachvollziehbar, warum immer noch oft die Schutzund Unterstützungsleistungen der Kinder- und Jugendhilfe diesen Minderjährigen verweigert werden, obwohl der $\mathbb{4} 42$ SGB VIII ihre strukturelle Schutzbedürftigkeit so klar benennt. 


\section{Anmerkungen}

(1) Zahlenangaben nach: Bernd Engelmann: Asylverfahren und kindgerechte Gestaltung, Zahlen und Fakten. In: Bundesverband UMF; BAGJAW: Fachpolitisches Forum: Verbesserung der Situation junger Flüchtlinge in der Bundesrepublik Deutschland, 2006, Seite 72.

(2) Vgl. auch Ausschuss für die Rechte des Kindes, allgemeine Bemerkung
Nr. 6 (2005): Behandlung unbegleiteter und von ihren Eltern getrennter Kinder außerhalb ihres Herkunftslandes (http://www.b-umf.de/pdf/generalcommentsdeutsch.test-star. pdf).

(3) Vgl. hierzu auch: Peter, Erich; Die Inobhutnahme unbegleiteter ausländischer Minderjähriger. Grundlegende Erläuterungen zur Neuregelung des $\mathbb{S}$ 42 Abs. 1 S. 1 Nr. 3 SGB VIII, in: Das Jugendamt, 02/2006, S. 60 ff.

(4) Peter, 2006, S. 61
(5) Bundestags-Drucksache 16/2633 vom 20.9.2006 . Antwort der Bundesregierung auf die Kleine Anfrage zum Umgang mit minderjährigen unbegleiteten Flüchtlingen

(6) Siehe auch BVG vom 8.7.2004 - 5 C 63.03 und vom 12.8.2004 - $5 \mathrm{C}$ 51.03.

(7) Entwurf eines Gesetzes zur Umsetzung aufenthalts- und asylrechtlicher Richtlinien der Europäischen Union, BT-Drucksache 16/5065 Art. 1. 38, c.

\section{Monitoring}

In der Rubrik »Monitoring « nehmen Autorinnen und Autoren einzelne Arbeits- und Themenfelder der Sozialen Arbeit in den Blick um zu untersuchen, was sich dort Neues tut oder warum dort Praxis und Theorie auf der Stelle treten. Die Artikel sind eine aktuelle Ergänzung zu den wechselnden Schwerpunktthemen aus der Sozialen Arbeit in den Blättern der Wohlfahrtspflege.

Eine Übersicht der bisherigen Beiträge in der Rubrik »Monitoring «:

Sorgenkind lokale Ökonomie

Ergebnisse der dritte Befragung in den Programmgebieten der »Sozialen Stadt«

Von Christa Böhme und Thomas Franke

Blätter der Woblfahrtspflege 5/2007

Orientierungslos, mutlos, machtlos

Die Idee der Bürgergesellschaft hat es noch nicht zum gesellschaftlichen Leitbild geschafft - Das liegt auch an uns selbst Von Stefan Nährlich

Blätter der Woblfabrtspflege 4/2007

Synergielösungen für Sozialräume

Plädoyer für Fusionen kleiner Träger

Von Armin Wöhrle

Blätter der Woblfahrtspflege 4/2007

Das Betreuungsgesetz droht zu scheitern

Erforderlich sind eine konsequenteProfessionalisierung, mehr Kompetenzen für die Betreuungsbehörden, obligatorische Sozialgutachten sowie eine regelmäßige Berichterstattung

Von Wolf Crefeld

Blätter der Woblfahrtspflege 3/2007

Wider die Individualisierung der Selbsthilfe

Karriere eines strapazierten Begriffs - Beispiel: Selbsthilfe Wohnungsloser

Von Stefan Gillich

Blätter der Woblfahrtspflege 3/2007

Auf der Suche nach der guten Praxis

Die Qualitätsentwicklung in der Sozialen Arbeit - ein Überblick

Von Andrea Buckley und Karl-Heinz Boeßenecker

Blätter der Woblfahrtspflege 2/2007

\section{Distanzierte Nähe}

Wie Wohlfahrtsverbände und traditionelle Milieus in Ostdeutschland (nicht) in Beziehung stehen

Von Peter-Georg Albrecht

Blätter der Woblfahrtspflege 2/2007
Was Fachkräfte wissen müssen

Der Qualifikationsrahmen Soziale Arbeit

Von Peter Buttner und Ulrich Bartosch

Blätter der Woblfahrtspflege 1/2007, Seite 28-30

Das Format mit Inhalt füllen

Interview mit Wolf Rainer Wendt zum »Qualifikationsrahmen Soziale Arbeit «

Blätter der Wohlfahrtspflege 1/2007, Seite 29

Eltern stützen, Kinder schützen

Nach den Gesetzesänderungen:

Wie zukunftsfest ist das Kinder- und Jugendhilferecht?

Von Johannes Münder

Blätter der Wohlfahrtspflege 1/2007, Seite 31-32

Alles wird zur Dienstleistung

Die wirkungsorientierte Finanzierung von Jugendhilfe

Von Benjamin Landes

Blätter der Wohlfahrtspflege 1/2007, Seite 33-34

Schulsozialarbeit braucht mehr Rechte

Ein Berufsfeld der Sozialen Arbeit zwischen Lerntradition und Lebenswelt

Von Susanne Hartmann-Hanff

Blätter der Wohlfahrtspflege 1/2007, Seite 35-36

Qualitätssicherung zivilisiert den Wettbewerb

System und Arbeitsfelder im Vergleich

Von Roland Schmidt

Blätter der Wohlfahrtspflege 6/2006, Seite 230-232

Indikatoren gesucht

Neue Ansätze zur Integrationssteuerung in Deutschland

Von Kristin Schwarze

Blätter der Wohlfahrtspflege 6/2006, Seite 233-234

Die armen Kinder

Junge Menschen als die unsichtbaren Verlierer von Hartz IV

Von Gerda Holz

Blätter der Wohlfahrtspflege 5/2006, Seite 193-195

Der Jahresbezugspreis beträgt 58,- Euro (für Studierende und arbeitslose Bezieher auf jährlichen Nachweis 29,- Euro).

Die Einzelhefte können zum Preis von 11,- Euro zuzüglich Versandkosten und Mehrwertsteuer bezogen werden bei:

Nomos Verlagsgesellschaft, 76520 Baden-Baden, Telefon 07221 2104-39, Fax 07221210443,

E-Mailhohmann@nomos.de 